\title{
Assistência à saúde bucal em residências geriátricas de Granada, Espanha e Santa Catarina, Brasil*
}

\author{
Oral health assistance in nursing homes of Granada, Spain and Santa Catarina, \\ Brazil

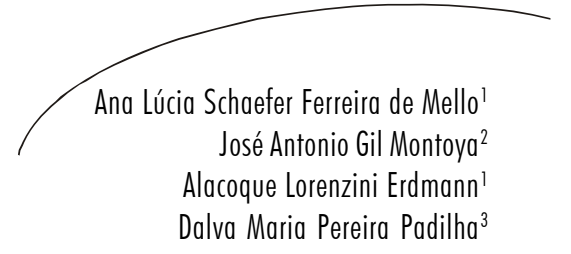

\section{Resumo}

Objetivo: Descrever a assistência à saúde bucal nas residências geriátricas da Província de Granada, Espanha, e Estado de Santa Catarina, Brasil, e comparar os dados obtidos. Metodologia: Trata-se de estudo exploratório, transversal. Os dados foram coletados por meio de questionário fechado, enviado pelo correio, para todas as residências geriátricas cadastradas em Granada (99) e Santa Catarina (78), em 2005. Resultados: O perfil das residências (29 em SC e 30 em Granada) foi semelhante, com predominância de instituições privadas. Não houve diferenças estatísticas significativas quanto aos residentes, exceto quanto ao número de instituições acima da mediana combinada para quantidade de mulheres e idosos totalmente dependentes, superiores em Granada. Embora as residências granadinas apresentassem maior número de profissionais da saúde, não houve diferenças quanto à insuficiente assistência à saúde bucal aos idosos, considerados: registro das condições de saúde bucal, circunstâncias em que essas condições são (re)avaliadas e realização sistemática de higiene bucal. Em face de um problema de saúde bucal apresentado pelo idoso, a família é comunicada, com maior frequência, nas residênci-

Palavras-chave: Idoso. Saúde Bucal. Assistência Odontológica para Idosos. Serviços de Saúde para Idosos. Instituição de Longa Permanência para Idosos. Estudo Comparativo. Estudos Transversais. Granada, Espanha. Santa Catarina, Brasil

Universidade Federal de Santa Catarina, Programa de Pós-graduação em Enfermagem. Florianópolis, SC, Brasil

2 Universidade de Granada, Faculdade de Odontologia. Granada, Espanha

3 Universidade Federal do Rio Grande do Sul, Programa de Pós-graduação em Odontologia. Porto Alegre, RS, Brasil

Estudo realizado no âmbito do Programa de Pós-graduação em Enfermagem da Universidade Federal de Santa Catarina - Brasil. Apoio CAPES - Coordenação de Aperfeiçoamento de Pessoal de Nível Superior, Brasil - Process: BEX 0555/04-5 e Universidade de Granada, Espanha

Correspondência / Correspondence

Ana Lúcia Schaefer Ferreira de Mello

Rua Rosa, 159, Pantanal

88040-270 - Florianópolis, SC, Brasil

E-mail: alfm@terra.com.br 
as granadinas. Conclusões: A assistência à saúde bucal não é prática sistemática nas instituições investigadas, em ambas as regiões, que transferem à família ou aos serviços locais de saúde o encaminhamento quando surgem problemas. Os procedimentos de higiene mais apontados foram escovação de dentes e próteses, porém práticas ineficazes são citadas. Foi frequente a alegação de falta de cooperação dos idosos para justificar a ausência de uma higiene bucal sistemática, revelando transferência de responsabilidades das instituições e cuidadores para o idoso.

\section{Abstract}

Objective: To describe and compare the oral health assistance at the Province of Granada, Spain, and at Santa Catarina, Brazil nursing homes. Methodology: It is a cross-sectional study. Data were collected through closed questionnaire, sent by mail, for the nursing homes registered in Granada (99) and Santa Catarina (78), in 2005. Results: The residences profile (29 in SC and 30 in Granada) was similar with predominance of the profit organizations. There were no significant statistical differences among the elderly, except concerning the average amount of nursing homes above the combined median values for number of women and totally dependent, higher in Granada. Although the grenadines residences present larger number of health professionals, there are no differences in precarious oral health assistance to the elderly, considering: the registration of oral health conditions, the circumstances in that those conditions are evaluated and the systematic oral hygiene protocol. In face of an oral health problem presented by the elderly, the family is communicated, more frequently, in the grenadine residences. Conclusions: Oral health assistance is not a systematic practice in the investigated institutions in both areas, and the solution is transfered to the elderly's family or local health services when problems appear. The most cited hygiene procedures were tooth and prostheses brushing, however ineffective practices are mentioned. It was frequent the allegation of lack of elderly's cooperation to justify the absence of a systematic oral hygiene, revealing the transference of responsibilities of the institutions and cares to the elderly.
Key words: Aged. Elderly. Nursing Homes. Oral Health. Dental Care for Aged. Health Services for the Aged. Homes for the Aged. Comparative Study. Cross-Sectional Studies. Granada, Espanha. Santa Catarina, Brasil

\section{INTRODUÇÃOO}

O cuidado à saúde bucal deve ser componente inseparável e articulado de qualquer sistema de cuidado integral à saúde do idoso, particularmente do idoso institucionalizado, bem como envolver a instituição na qual resida e os profissionais que nela trabalham, a família, os serviços públicos de saúde, a academia e o próprio idoso no exercício do autocuidado. ${ }^{1}$

No âmbito das ILPIs - Instituições de Longa Permanência para Idosos - costumeiramente são observadas omissões no campo da saúde bucal e relatos da literatura denunciam essas precárias condições nas mais diferentes realidades socioculturais. ${ }^{2-7}$ 
Tais fragilidades geralmente estão associadas à ausência de protocolos de procedimentos, falta de uma programação de assistência odontológica especializada, ausência de programas de orientação e capacitação dos cuidadores $^{8,9} \mathrm{e}$ insuficiente atenção do setor público. ${ }^{1}$ Acrescente-se, também, certo grau de desinformação e atitudes e concepções equivocadas em relação aos assuntos de saúde bucal entre os gestores e responsáveis pelas ILPIs, ${ }^{10}$ as quais quase sempre se defrontam com limitações financeiras e de infraestrutura. ${ }^{11}$

Entre os fatores que influenciam a qualidade da provisão do cuidado à saúde bucal em ILPIs, levando até à negligência, são citados: a falta de tempo dos cuidadores para dar conta do extenso rol de tarefas, contingente insuficiente de pessoal especializado, dificuldades físicas e de comportamento dos idosos e falta de programa de educação em saúde bucal para cuidadores de idosos. ${ }^{1,11}$
Envelhecimento, institucionalização e condições de saúde bucal dos idosos na Província de Granada e em Santa Catarina

Os dados demográficos apresentados no quadro I mostram a defasagem do Brasil e de Santa Catarina no processo de envelhecimento populacional, quando comparados com Espanha, Comunidade Autônoma de Andaluzia e sua Província de Granada. ${ }^{12-14}$ Todavia, a direção é a mesma. Avanços no campo da saúde, melhor circulação de informações, melhorias graduais no processo educacional e na distribuição da renda explicam a expressiva redução da taxa de fecundidade e a maior longevidade. Ressalta-se que a distribuição por gênero e zona de moradia tem padrão aproximado em ambos os países, com maior proporção feminina entre os idosos e concentração que se acentua nas zonas urbanas. Enquanto a diferença de expectativa de vida entre brasileiros e espanhóis é de aproximadamente oito anos, esse tempo é encurtado para quatro anos entre catarinenses e andaluzes. 
Quadro 1 - Dados demográficos: Brasil, Espanha, SC, Andaluzia e Granada. 2008

\begin{tabular}{|c|c|c|c|c|c|}
\hline & Brasil & Espanha & SC & Andaluzia & Granada \\
\hline \multicolumn{6}{|c|}{ Expectativa de vida ao nascer em 2005 (em anos) } \\
\hline Geral & 72,3 & 80,2 & 74,8 & 78,8 & 79,2 \\
\hline Mulheres & 76,1 & 83,5 & 78,1 & 82,1 & 82,3 \\
\hline Homens & 68,5 & 77,0 & 71,6 & 75,6 & 76,2 \\
\hline \multicolumn{6}{|c|}{ Expectativa de vida aos 60 anos (em anos) } \\
\hline Geral & 80,9 & 83,4 & 81,3 & 82,2 & - \\
\hline \multicolumn{6}{|c|}{ Expectativa de vida aos 65 anos (em anos) } \\
\hline Geral & 82,5 & 84,3 & - & 83,1 & - \\
\hline Mulheres & 83,7 & 86,1 & - & 84,9 & - \\
\hline Homens & 81,1 & 82,2 & - & 81,1 & - \\
\hline \multicolumn{6}{|c|}{ Expectativa de vida aos 70 anos (em anos) } \\
\hline Geral & 84,4 & 85,4 & - & 84,3 & - \\
\hline \multicolumn{6}{|c|}{ População de Idosos } \\
\hline $65 \mathrm{ou}+$ anos & $\begin{array}{c}\text { 11,5milhões } \\
(6,6 \%)\end{array}$ & $\begin{array}{c}7,2 \text { milhões } \\
(17 \%)\end{array}$ & $\begin{array}{c}349 \mathrm{mil} \\
(5,9 \%)\end{array}$ & $\begin{array}{c}\text { 1,17milhão } \\
(14,7 \%)\end{array}$ & $\begin{array}{l}133 \mathrm{mil} \\
(16,2 \%) \\
\end{array}$ \\
\hline $60 \mathrm{ou}+$ anos & $\begin{array}{c}\text { 16,7milhões } \\
(9,6 \%)\end{array}$ & $\begin{array}{c}9,3 \text { milhões } \\
(21,6 \%)\end{array}$ & $\begin{array}{c}539 \text { mil } \\
(9,2 \%)\end{array}$ & $\begin{array}{c}\text { 1,53milhão } \\
(19,2 \%)\end{array}$ & $\begin{array}{l}169 \mathrm{mil} \\
(20,6 \%)\end{array}$ \\
\hline \multicolumn{6}{|c|}{ Idosos - Mulheres } \\
\hline 65 ou + anos & - & $57,8 \%$ & - & $57,4 \%$ & $57,0 \%$ \\
\hline $60 \mathrm{ou}+$ anos & $55,9 \%$ & $56,4 \%$ & $55,0 \%$ & $56,1 \%$ & $56,1 \%$ \\
\hline $\begin{array}{l}\text { Idosos - zona } \\
\text { urbana }\end{array}$ & $83,4 \% * *$ & $88,5 \% * \% *$ & - & - & - \\
\hline
\end{tabular}

$* * 60$ ou + anos $\quad * * * 65$ ou + anos

Por conta das implicações nas políticas públicas, antes de prosseguir deve-se ressalvar que no Brasil são formalmente considerados "idosos" as pessoas que atingem a faixa etária dos 60 anos ou mais, e na Espanha, só após completarem 65 anos.

A maior longevidade tem sido acompanhada de elevado grau de dependência dos idosos, fato que traz consequências específicas ao campo da assistência à saúde bucal. $\mathrm{Na}$ Espanha, 32\% dos idosos apresentam algum tipo de incapacidade. Entre 65 e 79 anos, $21 \%$ e entre 80 anos ou mais, $59,8 \%$ revelam alguma dificuldade para realizar as atividades da vida diária. As dificuldades mais frequentes referem-se à mobilidade do corpo e de membros. No Brasil, entre os idosos com 65 anos ou mais, $18,4 \%$ indicavam dificuldade para alimentar-se, tomar banho e ir ao banheiro, $22 \%$ para caminhar cerca de 100 metros, $46 \%$ para caminhar mais de um quilômetro. ${ }^{15}$

Nesse contexto, ampliam-se as necessidades de serviços dirigidos aos idosos, que na Espanha compreendem: Atenção no Domicílio, Atenção Diurna (Centros-Dia) 
e os Serviços Residenciais - nestes, $60 \%$ dos idosos são dependentes. Os Serviços Residenciais ofereciam, em 2004, 266.392 vagas, correspondendo a uma cobertura de 3,6 vagas por 100 pessoas com 65 anos ou mais. O total de residências alcançava 4.888 , das quais $1.002(20,5 \%)$ eram públicas. As entidades privadas gerenciavam 75 de cada 100 vagas. Destas 75 vagas, 57 eram pagas integralmente pelo usuário e 18 eram disponibilizadas em regime de convênio, mediante financiamento com contribuição do setor público e coparticipação do usuário. O setor público operava por administração direta um quarto das vagas, as quais também eram financiadas em regime de copagamento, com contribuição do usuário. A maioria (60\%) das residências oferecia menos de 50 vagas e $25 \%$ dispunham de 50 a 100 vagas.

Não há levantamento sobre o universo das ILPIs brasileiras (cobertura, natureza ou financiamento). Estimativas apontam a existência de aproximadamente 19 mil idosos atendidos em instituições residenciais, mas o número deve ser maior se consideradas as instituições não cadastradas ou que funcionam na clandestinidade. ${ }^{16} \mathrm{O}$ setor público tem discretíssima presença como produtor desses serviços, que geralmente são oferecidos por entidades do terceiro setor, vinculadas a organizações religiosas, ou pela iniciativa privada com finalidade de lucro.

No plano normativo, no Brasil, a ILPI é uma modalidade de serviço de atendimento integral institucional ao idoso. Corres- ponde aos locais equipados para atender pessoas com 60 anos ou mais, sob regime de internato, mediante pagamento ou não, durante um período indeterminado, e cujas organizações devem dispor de recursos humanos para atender às necessidades de cuidados com a saúde, alimentação, higiene, repouso e lazer. ${ }^{17,18}$

No que tange à condição da saúde bucal dos idosos no Brasil, o mais recente levantamento epidemiológico nacional, o SB Brasil, ${ }^{19}$ revelou um CPO-D* entre os idosos de 27,8, em sua maior parte definido pelo componente perdido (93\%), seguido do cariado $(1,23 \%)$ e do obturado $(0,7 \%)$. Com relação à demanda por tratamento provocada pela doença cárie, $45,4 \%$ dos dentes necessitavam de exodontia. Quanto ao uso de próteses dentárias, 66,5\% e 31\% dos idosos possuíam-nas na arcada superior e inferior, respectivamente, e $32,4 \%$ e $56 \%$ necessitavam de próteses na arcada superior e inferior, respectivamente.

As condições da saúde bucal dos idosos que residem nas ILPIs brasileiras, apesar de não incluídas na amostra do SB Brasil, têm sido objeto de interesse de outros investigadores. Os relatos demonstram uma precária condição, representada por elevados índices de cárie e altas proporções de idosos edêntulos e de necessidades de próteses dentárias. $2,6,7,20$

Na Espanha, levantamento epidemiológico nacional examinou 3.460 pessoas aci-

Resultado da soma de dentes permanentes acometidos, restaurados ou perdidos por cárie dentária em um indivíduo. Pode variar de zero a 32. 
ma de 65 anos, institucionalizadas ou não. A saúde bucal foi considerada precária. Os resultados apontaram $31 \%$ de idosos edêntulos. $\mathrm{O}$ risco de desnutrição foi presente em $43 \%$ dos idosos edêntulos e $39 \%$ dos dentados e em $53 \%$ dos institucionalizados e 40\% dos não-institucionalizados. O índice CPO-D* atingiu 21,8. Com relação a hábitos de higiene bucal, $73 \%$ dos idosos dentados afirmaram escovar os dentes uma vez ao dia, ou menos. ${ }^{21}$ Em outro estudo, em 1998, foram avaliadas as condições de saúde bucal, exclusivamente dos idosos institucionalizados. Foram incluídas aleatoriamente 35 residências geriátricas, sendo examinados 3.282 idosos. A saúde bucal foi considerada ruim. O maior volume de tratamentos necessários (44,7\% dos idosos) era de ordem periodontal e constituía-se de procedimentos de baixo grau de complexidade, como instrução de higiene, remoção de placa e raspagem e alisamento radicular. ${ }^{22}$

Com relação ao padrão de utilização dos serviços odontológicos pelos idosos, em 1997, 16\% dos idosos espanhóis manifestaram ter visitado o dentista nos últimos seis meses, e em torno de $6,5 \%$ visitaram o profissional mais de uma vez nesse período. Por sua vez, no Brasil, dados de 1998 indicaram que $10,9 \%$ dos idosos visitaram o dentista no último ano, enquanto $7 \%$ nunca haviam consultado o dentista. Em Florianópolis, capital de Santa Catarina, pesquisa revelou que $10,7 \%$ dos idosos respondentes haviam consultado o dentista nos últimos três meses, enquanto $46,4 \%$ há muito tempo não procuravam o dentista. ${ }^{23,24}$
Este estudo pretende, então, contribuir para o melhor conhecimento dessa realidade, ao descrever e comparar a assistência à saúde bucal nas ILPIs da Província de Granada na Comunidade Autônoma da Andaluzia, Espanha e do Estado de Santa Catarina (SC), Brasil, respeitando-se o contexto e as especificidades locais.

\section{METODOLOGIA}

Trata-se de um estudo exploratório, transversal. Para a realização do levantamento na região de Andaluzia, Espanha, foi obtido o apoio da Consejería para la Igualdady Bienestar Social da Junta de Andalucía que forneceu a listagem com denominação e endereço das Residências Geriátricas de seu cadastro. Foram selecionadas as localizadas na província de Granada. Em Santa Catarina, deparou-se com deficiências no cadastramento das ILPIs, e a melhor opção foi a utilização de rol fornecido pelo Ministério Público Estadual.

Foi enviado por correio aos diretores das 99 residências da província de Granada constantes do cadastro oficial da Junta de Andalucía e das 78 ILPIs do Estado de Santa Catarina um envelope contendo: carta de apresentação, questionário (do tipo fechado - anexo 1) e um envelope selado para a devolução do instrumento aos pesquisadores. O questionário deveria ser respondido pelo dirigente ou outro profissional da saúde responsável pela instituição.

O questionário coletou informações sobre a natureza das instituições, disponibili- 
dade e qualificação dos recursos humanos, gênero e grau de dependência dos idosos, registro e avaliação das condições de saúde bucal, procedimentos de higiene bucal e encaminhamento para tratamento. A versão em espanhol, a primeira a ser confeccionada e aplicada, depois foi traduzida para o português. $\mathrm{Na}$ tradução foram feitas pequenas adaptações nas questões relativas à natureza jurídica das instituições e recursos humanos, atendendo às particularidades brasileiras.

Os dados foram organizados, tabulados e procedeu-se à análise estatística, que buscou comparar os diferentes aspectos investigados: características das residências, dos idosos residentes e da assistência em saúde bucal. Para averiguar a comparação das variáveis, foram utilizados os testes estatísticos não-paramétricos qui-quadrado e o teste da mediana, conforme Siegel e Caste11 an. ${ }^{25}$ Tomou-se o valor $\mathrm{p}<0,05$ como ponto de corte mínimo para verificação do nível de significância do teste.

\section{RESULTADOS}

A taxa de resposta foi considerada baixa. Nas instituições granadinas, obteve-se um percentual de resposta de 30,3\% (30 questionários) e nas catarinenses 37\% (29 questionários).

\section{Quanto às residências}

Em Santa Catarina, das 29 instituições respondentes, cinco eram de natureza privada não-filantrópica e 24 de natureza filantrópica (privada sem fins lucrativos). Não há ILPIs públicas em Santa Catarina. Em Granada, das 30 instituições respondentes, quatro eram públicas, 17 eram privadas sem vagas conveniadas com o setor público e nove, embora privadas, mantinham um conjunto de 314 vagas conveniadas com o setor público. Os respondentes de ambas as regiões apresentaram instituições de caráter privado e filantrópico, não havendo diferença estatística significativa.

A investigação sobre a frequência de profissionais da saúde, por ocupação, nas residências geriátricas é apresentada na tabela 1. Foram comparadas as ocupações equivalentes nas duas regiões. Não foi possível estimar a significância da diferença nas categorias psicólogo e ciurgião-dentista, pois o cálculo da frequência esperada para estimativa do qui-quadrado foi inferior a 5. A diferença entre as ocupações auxiliar/técnico de enfermagem, enfermeiro, médico e assistente social é significativa, contando as instituições granadinas com maior número de profissionais nessas áreas. Nenhuma das residências apresentou profissional de odontologia de nível médio (auxiliar de consultório dentário e técnico em higiene dental no Brasil ou bigienista dentalna Espanha). 
Tabela 1 - Frequência total do número de profissionais nas instituições, por ocupação. 2008.

\begin{tabular}{lcc}
\hline Ocupações & Santa Catarina & Granada \\
\hline Auxiliar/Técnico de Enfermagem** & 106 & 474 \\
Enfermeiro* $^{*}$ & 13 & 90 \\
Médico* & 16 & 27 \\
Psicólogo & 5 & 19 \\
Assistente Social* & 10 & 27 \\
Terapeuta Ocupacional* & 18 & 20 \\
Cirurgião-dentista & 1 & 2 \\
\hline
\end{tabular}

$* \mathrm{X}^{2}$ obtido $=323,37 ; \mathrm{X}^{2}$ tabelado $=20,00 ; \mathrm{gl}=4 ; \mathrm{p}>0,05$

Constatou-se que nem todas as residências dispunham de profissionais exercendo as ocupações investigadas. Em Santa Catarina, no entanto, a carência era mais acentuada. Em Santa Catarina, oito ILPIs, e em Granada uma ILPI não possuíam auxiliar de enfermagem, nem técnico de enfermagem. Em Santa Catarina, 15 não apontaram a presença de enfermeiro e 14 de médico. Já em Granada, quatro não apresentaram enfermeiro e oito não possuíam médi- co. Com relação à ocupação cirurgião-dentista, 26 residências em Santa Catarina e 28 em Granada não contavam com esse profissional no seu quadro.

Quanto aos idosos residentes

As características dos idosos residentes nas instituições respondentes, por gênero e grau de dependência, são apresentadas na tabela 2 .

Tabela 2 - Características dos idosos residentes nas Instituições de Longa Permanência. 2008.

\begin{tabular}{lcccccr}
\hline & \multicolumn{3}{c}{ Santa Catarina } & \multicolumn{3}{c}{ Granada } \\
\cline { 2 - 7 } & Mínimo & Máximo & Média (DP) & Mínimo & Máximo & Média (DP) \\
\hline Número de residentes** & 5 & 100 & $37,2(23,3)$ & 16 & 210 & $68,4(53,6)$ \\
Sexo masculino** & 0 & 40 & $12,4(10,2)$ & 0 & 93 & $23(23,2)$ \\
Sexo feminino* & 2 & 75 & $24,8(16,7)$ & 0 & 120 & $44(33,9)$ \\
Independentes** & 0 & 60 & $13,6(15,2)$ & 0 & 120 & $28,6(32,5)$ \\
Parcialmente dependentes*** & 0 & 45 & $13,7(12,3)$ & 0 & 90 & $22,7(26,3)$ \\
Tota lmente dependentes $* * *$ & 0 & 35 & $11,3(11,8)$ & 0 & 74 & $24,4(24,3)$ \\
\hline Para SC: $* \mathrm{n}=29 / * * \mathrm{n}=26$ & Para GR: $* \mathrm{n}=30 / * * \mathrm{n}=29$ & & & &
\end{tabular}


Em relação ao conjunto de todas as ILPIs investigadas, o valor da mediana combinada foi 41 para quantidade total de idosos, 12 para idosos do sexo masculino e 30 para idosos do sexo feminino. A comparação da quantidade de instituições, em SC e em Grana$\mathrm{da}$, acima e abaixo dos valores medianos combinados observados, revelou que somente a variável quantidade de idosos do sexo feminino apresentou diferença significativa $\left(\mathrm{X}^{2}\right.$ obtido $=9,44 ; \mathrm{X}^{2}$ tabelado $=9,14 ; \mathrm{gl}=1$; $\mathrm{p}<0,025)$. Neste caso, a quantidade de instituições apresentando valores acima da mediana combinada foram 18 em Granada e seis em SC.

Procedeu-se à mesma análise estatística com a variável nivel de dependência dos idosospara as Atividades da Vida Diária, decomposta em: Independente; Parcialmente Dependente; Totalmente Dependente. A mediana combinada foi 12 para quantidade de idosos independentes, 11 para quantidade de idososparcialmente dependentes e 14 para totalmente dependentes. A comparação da quantidade de instituições acima e abaixo das medianas combinadas calculadas indicou que somente no grau totalmente dependente a diferença foi significativa $\left(\mathrm{X}^{2} \mathrm{ob}-\right.$ tido $=4,62 ; \mathrm{X}^{2}$ tabelado $=3,84 ; \mathrm{gl}=1$; $\mathrm{p}<0,05)$. Observou-se que apresentaram valores acima da mediana combinada 17 residências granadinas e dez catarinenses.

\section{Quanto à assistência à saúde bucal em residências geriátricas}

Das ILPIs catarinenses, só oito afirmaram registrar as condições de saúde bucal dos idosos no momento da admissão, sete somente às vezes, e 14 relataram não registrar. Em Grana$\mathrm{da}$, só 12 afirmaram registrar, sete às vezese dez não registravam. A diferença entre regiões não apresentou significância estatística.

Outra questão investigada refere-se às circunstâncias em que eram avaliadas as condições de saúde bucal dos idosos. Apenas três ILPIs catarinenses e cinco granadinas afirmaram avaliar periodicamente pelo menos uma vez ao ano; tanto em Santa Catarina quanto em Granada, 16 ILPIs só o fazem quando existe algum problema/necessidade e nove não avaliam. A diferença estatística da variável investigada não foi significativa.

A tabela 3 apresenta as providências mais comumente tomadas no enfrentamento de um problema/necessidade de saúde bucal nos idosos. A análise da variável por elementos mostra que a diferença foi significativa apenas em relação ao item Avisa-se a um familiarpara que solucione um problema $\left(\mathrm{X}^{2}\right.$ obtido $=6,74 ; \mathrm{X}^{2}$ tabelado $=6,63 ; \mathrm{gl}=1$; $\mathrm{p}<0,01)$. 
Tabela 3 - Distribuição das frequências das providências tomadas frente à necessidade de assistência à saúde bucal dos idosos. 2008.

\begin{tabular}{lcc}
\hline & Santa Catarina & Granada \\
\hline Encaminha-se a uma Unidade de Saúde & 13 & 15 \\
Encaminha-se a um dentista (ou clínica) particular & 11 & 17 \\
É providenciado o atendimento pelo serviço odontológico da & 7 & 2 \\
residência & & 13 \\
Avisa-se a um familiar, para que solucione o problema* & 4 & 13 \\
\hline
\end{tabular}

$* \mathrm{X}^{2}$ obtido $=7,58 ; \mathrm{X}^{2}$ tabelado $=7,81 ; \mathrm{gl}=3 ; \mathrm{p}<0,01$

Também não foi significativa a diferença entre as regiões quando se tratou da higiene de dentes e próteses dentais nas instituições. Em Santa Catarina, 22 ILPIs afirmaram realizar procedimentos de higiene de dentes e próteses dentais de modo sistemático. Em Granada, esse número alcançou 21. Seis ILPIs catarinenses e sete granadinas procedem à higiene em alguns casos. Em am- bas as regiões, houve uma ILPI que informou não realizar a higiene sistemática de dentes e próteses.

A tabela 4 apresenta o levantamento dos procedimentos de bigiene de dentes e próteses dentais realizados nas duas regiões, tendo sido observadas diferenças estatísticas significantes no conjunto dos itens.

Tabela 4 - Distribuição das frequências em relação aos procedimentos de higiene bucal que são realizados nas instituições. 2008.

\begin{tabular}{lcc}
\hline & Santa Catarina & Granada \\
\hline Escovação de dentes* & 24 & 21 \\
Escovação de próteses* & 26 & 15 \\
Limpeza dos dentes ou próteses com gaze* & 11 & 8 \\
Enxágues das próteses* & 16 & 19 \\
Bochechos com soluções orais anti ssépticas* & 13 & 17 \\
Bochechos com água* & 14 & 5 \\
Uso de fio dental* & 7 & 0 \\
Estímulo/Motivação para os idosos realizarem sua própria & 1 & 9 \\
higiene dental & & \\
*X2 obtido $=12,60 ; \mathrm{X}^{2}$ tabelado $=12,59 ; \mathrm{gl}=6 ; \mathrm{p}<0,05$ & &
\end{tabular}


Quanto ao relato dos motivos da não-realização sistemática da bigiene bucal nos idosos residentes, também houve diferença estatística significativa no conjunto de itens apresentados na tabela 5 .

Tabela 5 - Distribuição das frequências dos itens que indicam o motivo de não ser realizada higiene bucal sistemática nos idosos nas instituições. 2008.

\begin{tabular}{lcc}
\hline & Santa Catarina & Granada \\
\hline Por não dispor de um dentista periodicamente na residência* & 7 & 5 \\
Falta de tempo dos profissionais cuidadores* & 7 & 5 \\
Falta de materiais ou equipamentos odontológicos* & 3 & 2 \\
Falta de conhecimentos sobre cuidados de saúde bucal de idosos* & 4 & 4 \\
Falta de treinamento prático em cuidados de saúde bucal de & 6 & 3 \\
idosos* & 7 & 8 \\
Pouca cooperação dos idosos* & 7 \\
\hline
\end{tabular}

$* \mathrm{X}^{2}$ obtido $=11,62 ; \mathrm{X}^{2}$ tabelado $=11,07 ; \mathrm{gl}=5 ; \mathrm{p}<0,05$

Metade das residências, tanto catarinenses quanto granadinas, relatou não ser suficiente a assistência odontológica disponível para cobrir as demandas/necessidades dos idosos.

\section{DISCUSSÃO}

Pesquisas de cunho clínico-epidemiológico na Espanha ${ }^{21,22}$ e no Brasil relatam que a saúde bucal de idosos residentes em ILPIs é bastante precária. Atribuem esse quadro à limitação de acesso aos serviços odontológicos e à fragilidade de uma rotina diária de higiene bucal. O presente estudo incidiu simultaneamente em duas regiões desses países e identificou em ambas deficiências no processo de cuidar da saúde bucal no âmbito das ILPIs.

Foi observada diferença estatística significante entre as instituições acima e abaixo do valor mediano dos residentes idosos do sexo feminino, bem como dos residentes considerados totalmente dependentes, maior em Granada do que em SC. A primeira observação deve estar associada à maior expectativa de vida das mulheres espanholas e a segunda pode estar associada ao critério de seleção de entrada no Brasil, onde as dificuldades financeiras levam muitas instituições a restringir o acesso aos idosos totalmente dependentes, preferindo ocupar-se dos demais.

Embora a proporção de profissionais de enfermagem seja maior nas residências de Granada, ainda assim não se observou diferencial relevante quanto aos cuidados em saúde bucal. As 59 ILPIs pesquisadas dispunham em seus quadros de apenas três dentistas e nenhum higienista dental/auxiliar de consultório dentário/técnico em higiene dental. Ainda que só a presença de cirurgião-dentista e respectivo pessoal auxi- 
liar não seja suficiente para solucionar os problemas de saúde bucal nas ILPIs, ${ }^{26}$ tais quantitativos indicam a fragilidade da assistência continuada à saúde bucal dos residentes. Portanto, é evidente a necessidade da difusão de conhecimentos nesta área entre todos os profissionais de saúde envolvidos no cuidado do idoso.

As normas de funcionamento de serviços de atenção ao idoso no Brasil, ${ }^{18}$ mais programáticas do que imperativas, indicam dois cirurgiões-dentistas nas ILPIs que se dediquem a abrigar idosos parcial ou totalmente dependentes, e dispensa dessa recomendação as ILPIs onde residam apenas idosos independentes. Parece ainda faltar um longo caminho até que se alcance a presença cotidiana desses profissionais, traduzindo-se na melhoria do cuidado à saúde bucal do idoso institucionalizado.

Em relação à SC, os idosos das residências granadinas dispõem de maior cobertura de profissionais da saúde, particularmente de enfermagem de nível superior e médio, ainda que insuficiências quantitativas na formação de equipe multiprofissional sejam observadas em ambas as regiões.

Embora as residências granadinas apresentem maior número de profissionais de saúde, não há diferenças estatísticas no que se refere à assistência à saúde bucal aos idosos, considerando-se o registro das condições de saúde bucal, as circunstâncias em que essas condições são avaliadas e reavaliadas e a realização sistemática de higiene bucal (dentes e próteses). Isto é relevante, pois se perde a oportunidade de di- agnosticar as necessidades iniciais de tratamento e ainda, ao longo da permanência, definir um plano de atenção às necessidades individuais de saúde bucal.

O padrão observado de realização sistemática de bigiene bucal não difere quando as regiões são comparadas: ainda há em ambas um contingente de instituições que não incorporara no seu cotidiano práticas de higiene bucal. Indagadas sobre que procedimentos de higiene bucal compunham sua prática cotidiana, as instituições catarinenses respondentes apresentaram maior frequência de procedimentos que as granadinas. Observou-se, contudo, que as respostas de ambos os grupos revelam a realização de procedimentos muitas vezes ineficazes, como o enxágue de próteses, bochechos com água ou a falta de atenção em procedimentos simples como motivação e reforço positivo para que o idoso realize o autocuidado.

Estudo realizado por Frenkel; Harvey; Newcombe ${ }^{8}$ sobre as práticas de cuidado à saúde bucal em 22 instituições de Avon (Inglaterra) também demonstrou que a maioria dos idosos necessita, mas não recebe, auxílio adequado às suas necessidades para a higiene bucal. No presente estudo, procedimentos básicos como escovação de dentes e de próteses totais não foram reportados por todas as instituições.

Os resultados do estudo confirmam que as ILPIs, confrontadas com as necessidades de assistência à saúde bucal, vão buscar para cada evento clínico uma solução externa, sem que haja uma abordagem coletiva e perma- 
nente. As instituições acreditam que os problemas bucais vivenciados pelos idosos são resolvidos via serviço público de saúde, rede privada ou por familiares, a quem se transfere o problema, fato este mais frequente nas residências geriátricas granadinas, segundo o estudo.

Nas urgências, algumas residências catarinenses reportaram atendimento clínico na própria instituição. Como não possuem profissionais em seus quadros, deduz-se que se socorrem do voluntariado eventual, comum em instituições filantrópicas brasileiras. ${ }^{17}$

A percepção da insuficiência na atenção à saúde bucal pode estar associada à incapacidade de mobilizar recursos financeiros suficientes para uma prestação mais qualificada de serviços, bem como de encontrar nos sistemas públicos assistência clínica pontual e de urgência.

As causas que impedem a provisão adequada de cuidado à saúde bucal estão relacionadas também a características organizacionais da instituição, formação profissional do cuidador, características pessoais do cuidador e características dos residentes. ${ }^{11}$ $\mathrm{O}$ inadequado cuidado à saúde bucal deve ser identificado e tratado como fenômeno de dimensão multifatorial, com implicações para educadores, gestores, profissionais da saúde e pesquisadores. ${ }^{28}$

Chung; Mojon e Budtz-Jorgensen verificaram certo grau de desinformação em relação aos assuntos de saúde bucal entre os responsáveis por organizar e fornecer cui- dado à saúde bucal em instituições suíças. Chalmers et al. ${ }^{29}$ investigaram os problemas encontrados na organização e provisão de cuidado à saúde bucal aos idosos de ILPIs de Adelaide (Austrália). Foram citados entre outros: desinteresse da família sobre a saúde bucal do seu idoso, problemas cognitivos e de comportamento dos idosos, limitações financeiras, pouca prioridade dada pelos cuidadores à saúde bucal, maior demanda de tempo para a tarefa, e o fato de os cuidadores não gostarem de realizar tais procedimentos. Algumas dessas situações também foram apontadas no presente estudo como motivo para que não se realizasse sistematicamente a higiene bucal dos idosos.

Embora tenha alcançado diferença estatisticamente significativa entre as regiões Granada e Santa Catarina, a pouca cooperação dos idosos parece ser afirmação recorrente, já que foi citada por todas as instituições que declaram não realizar a higiene bucal ou realizá-la somente em alguns casos. Esta questão precisa ser contextualizada, pois também pode refletir a tentativa de transferência de responsabilidades das instituições e cuidadores para a pessoa do idoso. O grau de comprometimento físico e/ou cognitivo do idoso é uma das muitas barreiras a serem superadas pelos responsáveis por fornecer a assistência à saúde bucal, o que requer motivá-lo para obter sua cooperação.

A atenção integral à saúde bucal para idosos institucionalizados apresenta-se ainda como desafio à sociedade. Esses desafios vão desde a superação da carência de pessoal capacitado para o cuidado, disponibi- 
lidade de equipamentos e materiais adequados, garantia de recursos financeiros para assistência, melhoria na capacidade gerencial e a consequente superação das precárias condições bucais. ${ }^{1,30}$

Estudo realizado por Pruksapong e MacEntee ${ }^{31}$ afirma que programas de saúde bucal em ILPIs devem ser implementados ou melhorados a partir da combinação de princípios de qualidade e avaliação em saúde que garantam a participação de todos os envolvidos, considerem os múltiplos atributos de qualidade, avaliem estrutura, processo/atividades e resultados do programa, utilizem métodos quanti-qualitativos de evidência e compartilhem conhecimentos e boas práticas.

Finalmente, como limitação importante deste estudo, aponta-se a baixa taxa de resposta, gerando a impossibilidade de generalização dos dados para as regiões, embora suficientes para a análise exploratória. Este fato pode ser reflexo do pequeno grau de importância dado ao tema da saúde bucal do idoso institucionalizado.

Acredita-se ser útil e meritório, devido ao caráter exploratório inicial que a pesquisa possui e às dificuldades de se coletar informações por meio do envio de questionário por correio, que sejam realizados mais estudos com outros desenhos e outras formas de coleta de dados, que incluam entrevistas, observação in loco e análise documental para compreensão mais aprofundada deste fenômeno.

\section{CONCLUSÕES}

A assistência à saúde bucal do idoso residente não é uma prática sistemática nas instituições investigadas, em ambas as regiões. A maioria das instituições não dispõe de registros das condições de saúde bucal dos idosos residentes, nem tem por hábito avaliar tais condições na admissão e durante o tempo de moradia.

Embora reconheçam ser insuficiente a assistência odontológica disponível, de um modo geral, as instituições tratam a saúde bucal do residente como questão exógena: a assistência é transferida pela comunicação dos casos à família ou aos serviços locais de saúde, quando surgem problemas.

Os procedimentos de higiene bucal apontados revelam ênfase na escovação de dentes e de próteses, ao tempo em que mostram a presença de práticas ineficazes. A frequente alegação de falta de cooperação dos idosos parece servir de escudo para justificar a ausência de uma higiene bucal sistemática naqueles dependentes para o cuidado à saúde bucal. Em todo caso, parece indicada a necessidade de serem difundidos conhecimentos sobre o cuidado à saúde bucal entre todos os profissionais que atuam nas ILPIs, bem como alcançar um maior envolvimento dos profissionais $\mathrm{da}$ odontologia.

Superar as iniquidades no acesso e proporcionar uma cobertura plena, capaz de preservar a saúde bucal do idoso institucio- 
nalizado, constituem responsabilidades coletivas, que extravasam a relação dual entre a instituição-residência e a pessoa residente e demandam uma apreciação sistêmica de um fenômeno complexo. Por isso, esperase que o presente estudo contribua não só para a compreensão dos modos como as ILPIs operam a assistência em saúde bucal em diferentes contextos, como para estimular a construção de melhores práticas de assistência à saúde bucal do idoso institucionalizado numa perspectiva mais ampla de cuidado integral da saúde para um envelhecimento saudável.

\section{AGRADECIMENTOS}

O desenvolvimento da pesquisa contou com o apoio do Departamento de Estomatologia e do Grupo de Investigação em Gerodontologia da Faculdade de Odontologia da Universidade de Granada e com a participação institucional da Conserjería para la Igualdady Bienestar Social da Junta de Andalucía.

\section{REFERÊNCIAS}

1. Mello ALSF, Erdmann AL. Unveiling contradictions and incorporating best practices in the elderly's oral health. Physis 2007 Jan/Apr; 17(1):139-56.

2. Reis SCGB, Higino MASP, Melo HMD, Freire MCM. Condição de saúde bucal de idosos institucionalizados em GoiâniaGO, 2003. Revista brasileira de epidemiologia 2005 Mar; 8(1): 67-73.

3. Henriksen BM, Ambjørnsen E, Laake K, Axéll TE. Oral hygiene and oral symptoms among the elderly in long-term care. Spec Care Dentist 2004 Sep/Oct; 24(5): 254-9.

4. Chalmers JM, Carter KD, Fuss JM, Spencer AJ, Hodge CP. Caries experience in existing and new nursing home residents in Adelaide, Austrália. Gerodontology 2002 Jul; 19(1): 30-40.

5. Montal S, Tramini P, Triay JA, Valcarcel J. Oral hygiene and the need for treatment of the dependent institutionalized elderly. Gerodontology 2006 Jun; 23(2):67-72.

6. Carneiro RMV, Silva DD, Sousa MLR, Wada RS. Saúde bucal de idosos institucionalizados, zona leste de São Paulo, Brasil, 1999. Cad Saude Publica 2005 dez; 21(6): 1709-16.

7. Gaiao LR, Almeida M, Heukelbach J. Perfil epidemiológico da cárie dentária, doença periodontal, uso e necessidade de prótese em idosos residentes em uma instituição na cidade de Fortaleza, Ceará. Revista brasileira de epidemiologia 2005 Set; 8(3): 316-23.

8. Frenkel H, Harvey I, Newcombe RG. Improving oral health in institutionalized elderly people by educating caregivers: a randomized controlled trial. Community Dent Oral Epidemiol 2001 Aug; 29(4): 28997.

9. Chung JP, Mojon P, Budtz-Jorgensen E. Dental care of elderly in nursing homes: perceptions of managers, nurses and physicians. Spec Care Dentist 2000 Jan/ Feb; 20(1): 12-7.

10. Logan HL, Ettinger R, McLeran H, Casko $\mathrm{R}$, Dal Secco D. Common misconceptions about oral health in the older adult: 
nursing practices. Spec Care Dentist 1991 Nov/Dec; 11(6): 243-7.

11. Chalmers JM, Levy SM, Buckwalter KC, Ettinger RL, Kambhu PP. Factors influencing nurses' aides' provision of oral care for nursing facility residents. Spec Care Dentist 1996 Mar/Apr; 16(2): 71-9.

12. Brasil. Ministério da Saúde. Datasus. Dados demográficos e sócio-econômicos do Estado de Santa Catarina. [acesso 2007 abr 22].Disponível em: URL: http:// tabnet.datasus.gov.br/cgi/ deftohtm.exe?ibge/cnv/popsc.def

13. Instituto Nacional de Estadísticas de España. Censo de Población y Viviendas 2001. [ acesso 2007 jul 05].Disponível em: URL: http://www.ine.es/ censo_accesible/es/inicio.jsp

14. Instituto Brasileiro de Geografia e Estatística. Tábuas completas de mortalidade, 2005. [acesso: $2006 \mathrm{abr}$ 25].Disponível em: URL: http:// www.ibge.gov.br/home/estatistica/ populacao/tabuadevida/2005/ defaulttab.shtm.

15. Instituto Brasileiro de Geografia e Estatística. Pesquisa Nacional por amostra domiciliar, 1998. [acesso 2006 ago 17] Disponível em: http:// www.ibge.gov.br/home/estatistica/ populacao/trabalhoerendimento/pnad98/ saude/acesso_utilizacao.shtm

16. Comissão de Direitos Humanos. Câmara dos Deputados. Relatório da V Caravana Nacional de Direitos Humanos: Sistema Asilar Brasileiro. Brasília, 2002.

17. Brasil. Ministério da Saúde. Portaria 1.395/GM de 10 de dezembro de 1999. Política Nacional de Saúde do Idoso. Brasília; 1999.

18. Brasil. Ministério da Previdência e Assistência Social. Portaria $n^{\circ} 73$ de 10 de maio de 2001. Normas de funcionamento de serviços de atenção ao idoso no Brasil. Brasília; 2001.

19. Brasil. Ministério da Saúde.Coordenação Nacional de Saúde Bucal. Projeto SB Brasil. Condições de saúde bucal da população brasileira 2002-2003. Resultados principais. Brasília; 2003.

20. Silva SRC, Valsecki Júnior A. Avaliação das condições de saúde bucal dos idosos em um município brasileiro. Rev Panam Salud Publica 2000 Oct; 8(4):268-71.

21. Spanish Geriatric Oral Health Research Group. Oral health issues of Spanish adults aged 65 and over. The Spanish Geriatric Oral Health Research Group. Int Dent J 2001 Jun; 51(3): 228-34.

22. Baciero GR, Gandarias FJG, Pérez LM. Oral health in the elderly in Spain. Bilbao: Eguía;1998.

23. Benedettti TRB, Petroski EL, Gonçalves LHT. Perfil do idoso do município de Florianópolis: relatório final. Florianópolis: Pallotti; 2004. 88p.

24. Benedetti TRB, Mello ALSF, Gonçalves LHT. Idosos de Florianópolis: autopercepção das condições de saúde bucal e utilização de serviços odontológicos. Ciência Saúde Coletiva 2007 dez; 12(6): 1683-90.

25. Siegel S, Castellan Jr. NJ. Estatística nãoparamétrica para ciências do comportamento. 2.ed. Porto Alegre: Artmed; 2006.

26. MacEntee MI. Oral care for successful aging in long-term care. J Public Health Dent 2000 Spring; 60(4): 326-9.

27. Selli L, Garrafa V. Solidariedade crítica e voluntariado orgânico: outra possibilidade de transformação societária. Hist Cienc Saude Manguinhos 2006; 13(2): 239-51. 
28. Fitzpatrick J. Oral health care needs of dependent older people: responsibilities of nurses and care staff. J Adv Nurs2000 Dec; 32(6): 1325-32.

29. Chalmers JM. Problems encountered with the organization and provision of dental care for Adelaide nursing home residents. In: Annual Meeting Of The American Association Of Public Health Dentistry, 62, 1999. Annals...Honolulu:
American Association of Public Health, 1999. p.37.

30. Strayer M. Oral health for homebound and institutionalized elderly. J Calif Dent Assoc1999 Sept; 27(9): 703-8.

31. Pruksapong M, Macentee MI. Quality of oral health services in residential care: towards an evaluation framework. Gerodontology 2007 Dec; 24(4): 224-30.

Recebido: 28/2/2008

Reapresentado: 06/8/2008

Aprovado: 21/10/2008 


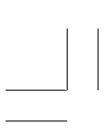

